九州大学学術情報リポジトリ

Kyushu University Institutional Repository

Molecular characteristics of colorectal neuroendocrine carcinoma; similarities with adenocarcinoma rather than neuroendocrine tumor

瀧澤，延喜

https://doi.org/10.15017/1654713

出版情報 : 九州大学，2015，博士（医学），課程博士

バージョン：

権利関係：やむを得ない事由により本文ファイル非公開（2） 
Elsevier Editorial System(tm) for Human Pathology

Manuscript Draft

Manuscript Number: YHUPA-D-15-00181R1

Title: Molecular characteristics of colorectal neuroendocrine carcinoma; similarities with adenocarcinoma rather than neuroendocrine tumor

Article Type: Original Article

Keywords: colorectal neuroendocrine carcinoma; neuroendocrine tumor; adenocarcinoma; Rb-p16 pathway; molecular characteristics

Corresponding Author: Dr. Yoshinao Oda, MD, PhD

Corresponding Author's Institution: Kyushu University, Graduate School of Med. Sciences

First Author: Nobuyoshi Takizawa, MD

Order of Authors: Nobuyoshi Takizawa, MD; Yoshihiro Ohishi, MD, PhD; Minako Hirahashi, MD, PhD; Shunsuke Takahashi, MD; Kazuhiko Nakamura, MD, PhD; Masao Tanaka, MD, PhD; Eiji Oki, MD, PhD; Ryoichi Takayanagi, MD, PhD; Yoshinao Oda, MD, PhD

Abstract: To further clarify the molecular features of colorectal neuroendocrine carcinomas (NECs), we immunohistochemically examined tumor samples from 25 NECs, including 9 small cell NECs (SCNECs) and 16 large cell NECs (LCNECs), 20 neuroendocrine tumors (NETs), and 21 poorly differentiated adenocarcinomas (PDCs) for the expression of several biomarkers (p53, $\beta$-catenin, Bcl-2, Rb, p16, p21, cyclinD1, and cyclinE), and used sequencing analysis to identify gene alterations of TP53, APC, CTNNB1, KRAS, and BRAF. The frequencies of aberrant p53 expression (88\%), $\beta$-catenin nuclear expression (48\%), and high expression of cyclinE (84\%) were significantly higher in NECs than in NETs $(0 \%, 5 \%$, and $5 \%, P<.01$, respectively). The immunohistochemical results of NECs and PDCs were similar. TP53, APC, KRAS, and BRAF gene mutations were variously detected in NECs and PDCs but not in any NETs. The frequencies of decreased expression of $\mathrm{Rb}(56 \%)$ and of high expression of $\mathrm{p} 16$ (56\%) and Bcl-2 (64\%) were significantly higher in NECs than in PDCs $(5 \%, 19 \%$, and 5\%, $\mathrm{P}<.05$, respectively) or NETs $(10 \%, 5 \%$, and $5 \%, \mathrm{P}<.01$, respectively). Such immunohistochemical characteristics of NECs were more evident in SCNECs than in LCNECs $(\mathrm{P}<.01)$. In conclusion, the molecular features of colorectal NECs are similar to those of adenocarcinomas and not to those of NETs. Decreased expression of Rb, and high expression of p16 and Bcl-2, are characteristics of NECs, suggesting that $\mathrm{Rb}-\mathrm{p} 16$ pathway disruption may contribute to the promotion of proliferative activity in colorectal NECs. SCNECs may be a prototype of NECs. 
Dr. R. V. Lloyd

Editor-in-Chief, Human Pathology

\section{Dear Dr. Lloyd:}

We are very grateful for your review of our manuscript. Please find enclosed our Responses to Reviewers and our revised manuscript entitled "Molecular characteristics of colorectal neuroendocrine carcinoma; similarities with adenocarcinoma rather than neuroendocrine tumor", which we hope will now be suitable for publication in Human Pathology.

Please address correspondence to Yoshinao Oda,

Department of Anatomic Pathology, Graduate School of

Medical Sciences, Kyushu University, 3-1-1 Maidashi, Higashi-ku, Fukuoka 812-8582, Japan

Tel: +81-92-642-6067, Fax: +81-92-642-5968,

E-mail: oda@surgpath.med.kyushu-u.ac.jp

Your kind consideration of our paper would be greatly appreciated.

Sincerely yours,

Yoshinao Oda, M.D. PhD.

Nobuyoshi Takizawa, M.D. 
Highlights

- We investigated the expression levels of a number of cancer-related proteins in colorectal neuroendocrine carcinomas, neuroendocrine tumors, and poorly differentiated adenocarcinomas using immunohistochemistry and mutation analysis.

- The molecular features of colorectal neuroendocrine carcinomas are similar to those of adenocarcinomas and not to those of neuroendocrine tumors.

- Decreased expression of $\mathrm{Rb}$ and high expression of $\mathrm{p} 16$ and $\mathrm{Bcl}-2$ are characteristics of colorectal neuroendocrine carcinomas.

- Rb-p16 pathway disruption may contribute to the promotion of proliferative activity in colorectal neuroendocrine carcinomas. 


\section{Responses to the editor's and reviewers' comments}

\section{Human Pathology}

Manuscript No.: YHUPA-D-15-00181

Title: Molecular characteristics of colorectal neuroendocrine carcinoma; similarities with adenocarcinoma rather than neuroendocrine tumor

Corresponding Author: Yoshinao Oda

\section{To the editor and reviewers:}

We are very grateful for your constructive comments and for the time you have taken to review our manuscript. Based on your comments, we have revised our manuscript, as follows.

\section{Editor's comments:}

1. Please include more cases if possible.

\section{Reply:}

Based on your comment, we have reviewed all the cases in our files. Unfortunately, we couldn't find more NEC cases suitable for this study. As described in our manuscript, we strictly selected the NEC cases according to a previous report and WHO classification. CD56 and NSE are not regarded as specific neuroendocrine markers. Furthermore, morphologically adenocarcinomatous tumors with some degree of chromogranin A/synaptophysin immunopositivities were also excluded.

2. Please carefully review the revised manuscript for syntax, grammar, and spelling before resubmission. If you are unsure where to find assistance with language editing, Elsevier offers such services at http://webshop.elsevier.com/. For example, "cut of" should be "cut-off" in table 1.

\section{Reply:}

We agree with the editor's comment. This revised manuscript was reviewed by KN International (http://www.kninter.com/) for syntax, grammar, and spelling.

We have added the following sentence. 
Page 18, lines 16-18

\section{Acknowledgment}

The English usage in this article was reviewed by KN International (http://www.kninter.com/).

3. Please include more quantitative, especially statistical, data in Abstract without exceeding 250-word limit. Also, please format the abstract per our journal style as a single paragraph without section headers.

\section{Reply:}

We agree with the editor's comment. We have corrected the manuscript as indicated. We have include more statistical data in the abstract within the space limit, and we have formatted the abstract as a single paragraph (See the revised abstract).

We have made the following corrections.

We have deleted this sentence from the abstract.

The molecular features of colorectal neuroendocrine carcinomas (NECs) haven't been well characterized.

Page 3 , line 2

To further clarify the molecular features of colorectal neuroendocrine carcinomas (NECs),

Page 3, lines 8-10

The frequencies of aberrant p53 expression (88\%), $\beta$-catenin nuclear expression (48\%), and high expression of cyclinE (84\%) were significantly higher in NECs than in NETs $(0 \%, 5 \%$, and $5 \%, \mathrm{P}<.01$, respectively).

Page 3, lines 10, 11

The immunohistochemical results of NECs and PDCs were similar.

Page 3, lines 13-15 
The frequencies of decreased expression of $\mathrm{Rb}(56 \%)$ and of high expression of $\mathrm{p} 16$ (56\%) and Bcl-2 (64\%) were significantly higher in NECs than in PDCs $(5 \%, 19 \%$, and $5 \%, \mathrm{P}<.05$, respectively) or NETs $(10 \%, 5 \%$, and $5 \%, \mathrm{P}<.01$, respectively).

Page 3, lines 16, 17

Such immunohistochemical characteristics of NECs were more evident in SCNECs than in LCNECs $(\mathrm{P}<.01)$.

Page 3, lines 18, 19

In conclusion, the molecular features of colorectal NECs are similar to those of adenocarcinomas and not to those of NETs.

Page 4, lines 1, 2

$\underline{\mathrm{Rb}}$-p16 pathway disruption may contribute to the promotion of proliferative activity in colorectal NECs.

Page 4, line 2, 3

SCNECs may be a prototype of NECs.

4. Please reduce the total number of tables and figures to a combined maximum of

8. This may be accomplished by condensing some items together, such as tables 3 and 4 . Alternatively, less important visual aids can be designated as supplementary materials that will be published online only, or else deleted.

\section{Reply:}

We agree with the editor's comments. We have reduced the total number of tables and figures as follows. We combined previous tables 3 and 4 into revised table 1 . In addition, we designated previous tables 1 and 2 as revised supplementary tables 1 and 2 .

Previous Table $1 \rightarrow$ Revised Supplementary table 1

Previous Table $2 \rightarrow$ Revised Supplementary table 2

Previous Table 3, Table $4 \rightarrow$ Revised Table 1

Previous Table $5 \rightarrow$ Revised Table 2

Previous Table $6 \rightarrow$ Revised Table 3 
Previous Table $7 \rightarrow$ Revised Table 4

Previous Table $8 \rightarrow$ Revised Table 5

5. Panel labels in figures $1(\mathrm{~A}-\mathrm{F})$ and $2(\mathrm{~A}-\mathrm{H})$ should consist of stand-alone capital without the white square backgrounds. All abbreviations in the tables (eg, Tm, PCR, NEC, PDC, SC, LC, UICC, ENETS) should be defined in accompanying footnotes with the "Abbreviations:" header. Other footnotes should be labeled with superscript letters not symbols. Note in table 7 that if the column head already indicates the number of it is not necessary to repeat the same number in the data cells.

\section{Reply:}

We agree with the editor's comment. We corrected panel labels in figures 1 (A-F) and 2 (A-H) as stand-alone capital letters without the white square backgrounds (See the revised figures 1 and 2).

We added an "Abbreviations:" heading in each table and figure legend (See the revised tables and figure legends). We labeled other footnotes with superscript letters not We corrected previous table 7 as suggested (See the revised table 4).

6. The addition of a running head (condensed version of full title limited to 60 and funding disclosure on the title page would be helpful.

\section{Reply:}

We agree with the editor's comment. We have added a running title and funding disclosures on the title page as follows.

Page 2, lines 9-11

Running title: Molecular characteristics of colorectal neuroendocrine carcinoma

\section{Disclosures:}

The authors declare that there are no conflicts of interest or funding disclosures to make.

Reviewers' comments:

Reviewer \#1: 
1) The paper needs English language improvement.

\section{Reply:}

We agree with the reviewer's comment. This revised manuscript was reviewed by KN International (http://www.kninter.com/) for syntax, grammar, and spelling.

We have added the following sentence.

Page 18, lines 16-18

\section{Acknowledgment}

The English usage in this article was reviewed by KN International (http://www.kninter.com/).

2) The authors should better underline if there are differences in terms of clinicopathological and/or immunohistochemical/molecular features between NETs (G1 versus G2).

\section{Reply:}

We agree with the reviewer's comment.

NETs showing larger tumor size $(\geqq 3 \mathrm{~cm})$, mitotic activity, advanced stage, lymph node metastasis, and venous invasion corresponded to NET G2 as shown in the following table. 
Clinical and histological characteristics of colorectal NET G1 and NET G2

\begin{tabular}{|c|c|c|c|}
\hline & $\begin{array}{l}\text { NET G1 } \\
n=18\end{array}$ & NET G2 case1 & NET G2 case 2 \\
\hline Age $(y)$ & 56 (median) & 69 & 57 \\
\hline Sex & 11 Male: 7 Female & Male & Male \\
\hline Tumor location & Rectum & Rectum & Rectum \\
\hline Tumor size $(\mathrm{cm})$ & 0.75 (median) & 3 & 3.8 \\
\hline Lymph node metastasis & $0 / 18$ & + & + \\
\hline Distant metastasis & $0 / 18$ & - & - \\
\hline \multicolumn{4}{|l|}{ Tumor stage } \\
\hline (UICC) & 118 & IIIB & IIIB \\
\hline (ENETS) & IA 15, IB 3 & IIIB & IIIB \\
\hline \multicolumn{4}{|l|}{ Neuroendocrine immunophenotype } \\
\hline Chromogranin A & $13 / 18$ & + & + \\
\hline Synaptophysin & $16 / 18$ & + & + \\
\hline Necrosis & $0 / 18$ & - & - \\
\hline Mitosis /10HPF & 0 (median) & 0 & 2 \\
\hline Lymphatic permeation & $0 / 18$ & - & - \\
\hline Venous invasion & $0 / 18$ & + & - \\
\hline Tumor-infiltrating lymphocytes & $0 / 18$ & - & - \\
\hline Focal squamous differentiation & $0 / 18$ & - & - \\
\hline Intracytoplasmic mucin & $0 / 18$ & - & - \\
\hline \multicolumn{4}{|l|}{ Additional components } \\
\hline Adenoma or Adenocarcinoma & $0 / 18$ & - & - \\
\hline
\end{tabular}

Abbreviations: NET, neuroendocrine tumor; UICC, Union for International Cancer Control; ENETS, European Neuroendocrine Tumor Society; L.I., labeling index; HPF, high-power field 
Immunophenotypes in NET G1 were similar to those in NET G2, as shown in this table.

Immunohistochemical characteristics of colorectal NET G1 and NET G2

\begin{tabular}{lccc}
\hline & $\begin{array}{c}\text { NET G1 } \\
\mathrm{n}=18\end{array}$ & NET G2 case1 & NET G2 case2 \\
\hline Loss of expression of $\mathrm{Rb}$ & $2 / 18$ & - & - \\
High expression of $\mathrm{p} 16$ & $0 / 18$ & - & + \\
High expression of bcl-2 & $1 / 18$ & - & - \\
Aberrant expression of p53 & $0 / 18$ & + & - \\
High expression of p21 & $7 / 18$ & - & - \\
High expression of cyclinE & $1 / 18$ & - & - \\
Nuclear expression of $\beta$-catenin & $1 / 18$ & + & + \\
High expression of cyclinD1 & $18 / 18$ & & + \\
\hline
\end{tabular}

Abbreviations: NET, neuroendocrine tumor

We have added the following sentences.

Page 10, lines 17, 18

The 2 cases of NET G2 were graded by Ki-67 L.I. (5\% and 4.5\%, respectively) based on the definition in the WHO classification [1].

Page 11, lines 11, 12

NETs showing larger tumor size $(\geqq 3 \mathrm{~cm})$, advanced stage, and lymph node metastasis corresponded to NET G2.

Page 12, line 5

In the NETs, venous invasion and mitotic activity were seen only in cases of NET G2.

Page 12, line 15

Immunophenotypes in NET G1 were similar to those in NET G2. 
3) Based on their findings, the authors should better comment on whether SCNECs and LCNECs should remain separated or be unified into a high-grade category, or if the latter tumor should be better incorporated into PDCs.

\section{Reply:}

We agree with the reviewer's comment.

Patients with NECs showed the statistically shortest overall survival period among the three groups (NECs, NETs, and PDCs), as shown in the following figure A. No significant prognostic difference was seen between SCNECs and LCNECs, as shown in the following figure B. From a prognostic standpoint, SCNECs and LCNECs can be unified into a high-grade category.

However, from the standpoint of pathological classification, the morphological features of SCNECs and LCNECs differ significantly by definition. Furthermore, there are some differences in molecular features between them. In this study, we believe that SCNECs and LCNECs should remain separated from the standpoint of pathological classification.

Futhermore, we believe that LCNECs should not be readily incorporated into PDCs because LCNECs show 1) unequivocal neuroendocrine morphology and immunophenotype in contrast to PDCs, and 2) significantly worse prognosis than PDCs, as shown in the following figure $\mathrm{C}$, even though LCNECs have some similar molecular features with PDCs. 
A

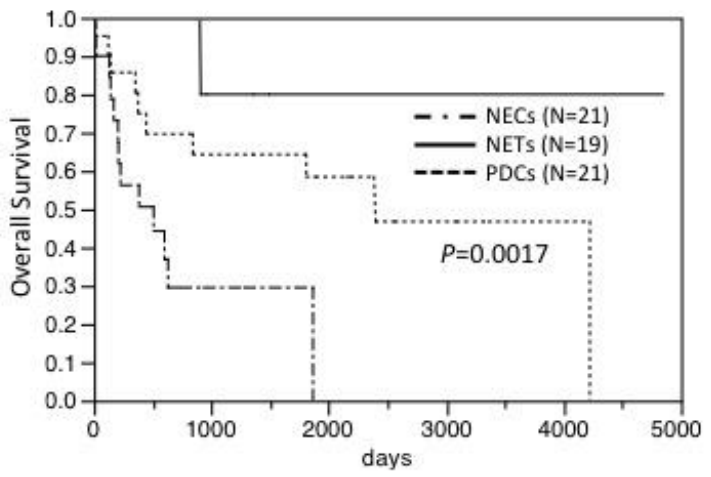

C

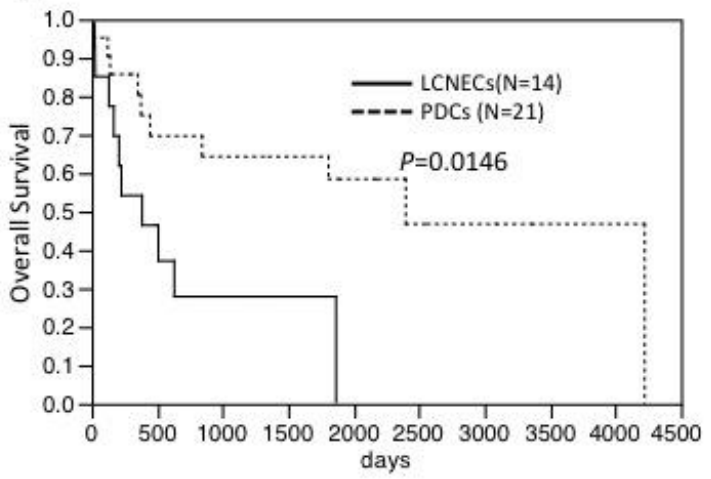

B

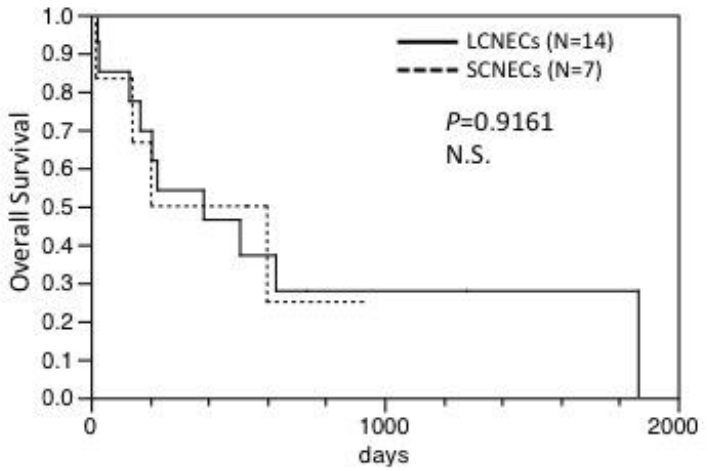

Kaplan-Meier methods $P<0.05$, Log-rank test

We have added sentences as follows.

Page 17, lines 1-12

In this study, patients with NECs showed the statistically shortest overall survival period among NECs, NETs, and PDCs (data not shown). No significant prognostic difference was seen in between SCNECs and LCNECs. From the standpoint of prognosis, SCNECs and LCNECs can be unified into a high-grade category. However, from the standpoint of pathological classification, the morphological features of SCNECs and LCNECs differ significantly by definition. Furthermore, there are some differences in molecular features between them. In this context, we believe that $\underline{\text { SCNECs and LCNECs should remain separate pathological categories. }}$ Furthermore, we believe that LCNECs should not be readily incorporated into PDCs, because LCNECs show 1) unequivocal neuroendocrine morphology and immunophenotype in contrast to PDCs, and 2) significantly worse prognosis than PDCs (data not shown), even though LCNECs have some similar molecular features with PDCs. 
4) The authors found that Bcl-2 expression level in LCNECs was significantly higher than that in PDCs $(\mathrm{P}=.0084)$. Can this immunohistochemical marker be useful in the differential diagnosis between LCNEC and PDC on biopsy? Please, comment on the immunohistochemical profile obtained in the two cases of NECs diagnosed on biopsy. In this regard, have the authors the possibility of comparing bioptic material of their series cases, namely bioptic immunohistochemical profiles of LCNECs versus PDCs?

\section{Reply:}

We thank you for your constructive comment.

In this study, the two bioptic samples were SCNEC and LCNEC, respectively. We are sorry for missing some immunohistochemical data on the bioptic LCNEC sample from the statistical analysis in the previous manuscript, by our own mistake. We have corrected the immunohistochemical data and the results of the statistical analysis in the revised manuscript and revised table 2 .

The bioptic immunohistochemical profiles of SCNEC and LCNEC are presented in the following table.

Immunohistochemical characteristics of bioptic samples of colorectal NECs

\section{SCNEC LCNEC}

Loss of expression of $R b$

High expression of $\mathrm{p} 16$

High expression of bcl-2

Aberrant expression of $p 53$

High expression of p21

High expression of cyclinE

Nuclear expression of $\beta$-catenin

High expression of cyclinD1

Abbreviations: NEC, neuroendocrine carcinoma; SCNEC, small cell neuroendocrine carcinoma; LCNEC, large cell neuroendocrine carcinoma 
The characteristic molecular features of NECs (loss of Rb expression and high expression of p16 and Bcl-2) are shown even in the 2 cases of NEC diagnosed on biopsy.

Based on the corrected data, the frequencies of high expression of Bcl-2 and of loss of $\mathrm{Rb}$ nuclear expression were significantly higher in LCNECs than in PDCs $(\mathrm{P}=.0043$, $\mathrm{P}=.0303$, respectively).

However, we think that the diagnostic utility of $\mathrm{Bcl}-2$ and $\mathrm{Rb}$ immunohistochemistry is limited for the differential diagnosis between LCNECs and PDCs, since most PDCs and more than half of LCNECs lack high expression of Bcl-2 and loss of Rb expression (revised table 2).

Based on the reviewer's comments, we have further examined the bioptic samples of 4 LCNECs and 9 PDCs, for the expression of Bcl-2 and Rb. As a result, high expression of Bcl-2 was not observed in any LCNECs (0/4) or PDCs (0/9). Loss of Rb expression was not observed in any LCNECs (0/4) or in most PDCs (1/9). The results of these bioptic samples were mostly concordant with those of surgical specimens.

Immunohistochemical results of additional 4 LCNECs and 9 PDCs using biopsy specimens

\begin{tabular}{cccc}
\hline & & High expression of Bcl-2 & Loss of Rb expression \\
\hline LCNEC & 1 & - & - \\
& 2 & - & - \\
& 3 & - & - \\
PDC & 4 & - & - \\
& 1 & - & - \\
& 2 & - & - \\
& 3 & - & - \\
& 4 & - & - \\
& 5 & - & - \\
6 & - & - \\
7 & - & - \\
8 & - & - \\
9 & - & - \\
\hline
\end{tabular}

Abbreviations: LCNEC, large cell nuroendocrine carcinoma;

PDC, poorly differentiated adenocarcinoma 
It appears that $\mathrm{Bcl}-2 / \mathrm{Rb}$ immunohistochemistry is not useful in the differential diagnosis between LCNEC and PDC on biopsy.

We think that the careful examination of morphological features on HE slides and the confirmation of neuroendocrine immunophenotype (positive staining of chromogranin A or synaptophysin) are paramount for the differential diagnosis between LCNECs and PDCs.

We have added the following sentences.

Page 13, lines 1, 2

These characteristic molecular features of NECs (loss of $\mathrm{Rb}$ expression and high expression of p16 and Bcl-2) were shown even in the 2 cases of NEC diagnosed on biopsy.

Page 13, lines 5, 6

The frequencies of loss of $\mathrm{Rb}$ expression and of high expression of $\mathrm{p} 16$ and $\mathrm{Bcl}-2$ were significantly higher in SCNECs than in LCNECs $(P=.0009 ; P=.0009 ; P=.0049$, respectively).

Page 13, lines 7, 8

The expression levels of Rb, p16, and Bcl-2 of LCNECs were intermediate between those of SCNECs and those of PDCs (Table 2).

Page 17, line 13 - Page 18, line 5

In this study, the frequencies of high expression of $\mathrm{Bcl}-2$ and of the loss of $\mathrm{Rb}$ nuclear expression were significantly higher in LCNECs than in PDCs. We further examined the bioptic samples taken from 4 cases of LCNECs and 9 cases of PDCs, for the expression of Bcl-2 and Rb. Unfortunately, we did not observe high expression of Bcl-2 or the loss of Rb expression in any LCNECs or in most PDCs $(0 / 9,1 / 9$, respectively). The results of these bioptic samples were mostly concordant with those of surgical specimens. 
In this context, we think there is limited diagnostic utility of Bcl-2 and $\mathrm{Rb}$

immunohistochemistry for the differential diagnosis between LCNECs and PDCs, since most PDCs and more than half of LCNECs lack high expression of Bcl-2 and loss of Rb expression (Table 2).

We think that the careful examination of morphological features on HE slides and the confirmation of neuroendocrine immunophenotype (positive staining of chromogranin A or synaptophysin) are paramount for the differential diagnosis between LCNECs and PDCs.

Reviewer \#2: Takizawa et al. studied a large series of neuroendocrine tumors in colorectal systems by immunohistochemistry and mutational analysis of cancer-related genes. They found commonalities of NEC cases to those found in adenocarcinoma and concluded that NEC may be closer to adenocarcinoma rather than NET.

Although the data themselves are of interest, the comparison between NEC and NET is problematic and conclusion thereby is difficult to follow. The authors should better concentrate on characterization of molecular expressions in NEC and NET, and discuss the similarity and dissimilarity between NEC and adenocarcinoma without speculation of tumor origin.

The data of molecular expressions should be presented in a more systematic manner.

1. Although the data themselves are of interest, the comparison between NEC and NET is problematic and conclusion thereby is difficult to follow. The authors should better concentrate on characterization of molecular expressions in NEC and NET, and discuss the similarity and dissimilarity between NEC and adenocarcinoma without speculation of tumor origin.

\section{Reply:}

We agree with the reviewer's comments. We have attempted to concentrate on the characterization of molecular expression in NEC and NET, and to discuss the similarities and dissimilarities between NEC and adenocarcinoma. We have also excluded the term "histogenetically" from the revised manuscript.

Page 4, lines 1, 2 
$\underline{\mathrm{Rb}}$-p16 pathway disruption may contribute to the promotion of proliferative activity in colorectal NECs.

Page 11, line 15 - Page 12, line 2

Compared to NETs, NECs showed significantly more frequent lymphatic permeation (NECs $65 \%, P<.0001$ ), venous invasion (NECs 83\%, $P<.0001$ ), and necrosis (NECs $\underline{84 \%, P<.0001)}$.

The frequency of the presence of intracytoplasmic mucin and that of tumor-infiltrating lymphocytes of NECs (16\% and 8\%, respectively) were significantly lower than those of PDCs $(P=.0002$ and $P=0.02$, respectively).

Mitotic counts were significantly higher in NECs (median 29/10HPF) than in NETs (median $0 ; P<.0001)$ or PDCs (median 12; $P=0.01$ ).

Page 12, lines 10-14

Compared to NETs, NECs showed significantly higher frequencies of aberrant p53 (NECs 88\%, $\mathrm{P}<.0001$ ) and $\beta$-catenin nuclear expression (NECs 48\%, $\mathrm{P}=.0016)$ and higher expression of cyclinE (NECs 84\%, $\mathrm{P}<.0001$ ).

With regard to the expression of those markers, there were no statistical differences between NECs and PDCs.

Page 15, lines 12-14

These immunohistochemical and molecular genetic features strongly substantiate the general concept that the molecular characteristics of colorectal NECs are similar to those of adenocarcinomas but not to those of NETs.

Page 15, lines 15, 16

On the other hand, we demonstrated that low expression of $\mathrm{Rb}$ and high expression of p16 and Bcl-2 were frequent in colorectal NECs.

Page 15, line 19 - Page 16, line 2

Thus, it has been speculated that Rb-p16 pathway disruption may contribute to the promotion of proliferative activity in NECs. 
Page 16, lines 2, 3

Our results further substantiate the fact that Rb-p16 disruption can be applicable to the high proliferative activity of colorectal NECs.

Page 18, lines 14, 15

SCNECs may be a prototype of NECs, whereas the molecular features of LCNECs are in between those of SCNECs and those of PDCs.

2. The data of molecular expressions should be presented in a more systematic manner.

\section{Reply:}

We agree with the reviewer's comments. We have subheaded each paragraph in "Results 3.4" as follows (See the revised manuscript).

Page 12, line 9

The differences and similarities of molecular characteristics among NECs, NETs, and PDCs.

Page 12, line 16

Molecular characteristics of NECS.

Page 13, line 3

The differences and similarities of molecular characteristics between SCNECs and LCNECs.

Page 13, line 9

The molecular characteristics of NEC components in combined NECs.

Page 13, line 14

Correlations of expression levels of molecular markers of NECs.

\section{Complement:}

1. We have corrected "Highlights" as follows on the basis of the above revision.

- The molecular features of colorectal neuroendocrine carcinomas (NECs) haven't been well characterized.

$\rightarrow$ We have deleted this sentence. 
- The colorectal NECs may be histogenetically closer to adenocarcinoma than to NETs. $\rightarrow$ The molecular features of colorectal neuroendocrine carcinomas are similar to those of adenocarcinomas and not to those of neuroendocrine tumors.

- Rb-p16 pathway disruption may contribute to the histogenesis of colorectal NECs. $\rightarrow \cdot \mathrm{Rb}$-p16 pathway disruption may contribute to the promotion of proliferative activity in colorectal neuroendocrine carcinomas.

2. We have moved the following sentence, which was written in the footnote of the previous table 4 , to 2.3 Histological assessment in the revised manuscript.

Page 8, lines 5-7

With regard to the 2 cases for which only biopsy specimens were available, the analyses for lymphovascular invasion, squamous differentiation, and the presence or absence of additional non-NEC components could not be performed.

3. We have deleted the following sentences in order to simplify the data in this study.

In the previous Results 3.4 (Page 13, line 6).

MLH1 was lost in PDCs but not in NETs and NECs.

In the previous Discussion (Page 16, lines 15, 16).

The Wnt pathway may occasionally contribute to neuroendocrine differentiation from colorectal adenoma/adenocarcinoma.

We also deleted the values of MLH1 and MSH2 expression from the revised table 2.

4. We have added the following sentence in Results 3.5.

Page 14, lines 11, 12

APC gene mutation was observed in only 1 case of NEC and 5 cases of PDCs. CTNNB1 mutations were observed in a small population of NETs, NECs, and PDCs (Table 4).

5. The range of age in NETs was incorrect in previous table 3. We have corrected it as 
follows (See the revised table 1).

$\underline{46-85 \rightarrow 24-86 .}$

6. The percentage of chromogranin A-positive cases in NETs was incorrect in previous table 3. We have corrected it as follows (See the revised table 1).

$\underline{76 \rightarrow 75 \text {. }}$

7. The number of $\%$ abnormality of p21 in NETs was incorrect in previous table 5. We have corrected it as follows (See the revised table 2).

$\underline{50 \rightarrow 40 .}$

8. The $P$-values of the correlations between the expression of some molecular markers were incorrect in the previous manuscript. We have corrected them as follows.

Page 13, line 17

$(\underline{P=.0128)}$

Page 13, line 19

$(\underline{P=.0246})$

9. We have corrected the statistical data as follows. See the revised results and tables.

In the revised manuscript:

Page 12 , line $18 \underline{(P=.0014 ; P=.0003 ; P<.0001, \text { respectively })}$

Page 12, line $19 \underline{(P=.0002 ; P=.0105 ; P<.0001, \text { respectively })}$

Page 13 line $16(\underline{P=.0002})$

Page 13, line $18 \underline{(P=.0295)}$

Page 14, line 19 - Page 15, line 1

For the first time, we demonstrated that the expression levels of cyclinE in NECs were similar to those in PDCs and higher than those in NETs.

Page 15, lines 1-3

High expression of cyclinE, as well as aberrant p53 and nuclear $\beta$-catenin expression, appear to be common features of colorectal NECs and PDCs but not of NETs. 
In the revised table 2:

We have underlined the corrected numbers in the revised table 2. 
1 Molecular characteristics of colorectal neuroendocrine carcinoma; similarities with

2 adenocarcinoma rather than neuroendocrine tumor

3

4 Nobuyoshi Takizawa $\mathrm{MD}^{\mathrm{a}}$, Yoshihiro Ohishi MD, $\mathrm{PhD}^{\mathrm{a}}$, Minako Hirahashi MD, $\mathrm{PhD}^{\mathrm{a}}$,

$5 \quad$ Shunsuke Takahashi $\mathrm{MD}^{\mathrm{a}}$, Kazuhiko Nakamura MD, $\mathrm{PhD}^{\mathrm{b}}$, Masao Tanaka, $\mathrm{MD}, \mathrm{PhD}^{\mathrm{c}}$, Eiji

6 Oki MD, $\mathrm{PhD}^{\mathrm{d}}$, Ryoichi Takayanagi MD, $\mathrm{PhD}^{\mathrm{b}}$, Yoshinao Oda MD, $\mathrm{PhD}^{\mathrm{a}}$

7

$8{ }^{\mathrm{a}}$ Department of Anatomic Pathology, Graduate School of Medical Sciences, Kyushu

9 University, Higashi-ku, Fukuoka, Japan

$10{ }^{\mathrm{b}}$ Department of Medicine and Bioregulatory Science, Graduate School of Medical Sciences,

11 Kyushu University, Higashi-ku, Fukuoka, Japan

$12{ }^{\mathrm{c}}$ Department of Surgery and Oncology, Graduate School of Medical Sciences, Kyushu

13 University, Higashi-ku, Fukuoka, Japan

$14{ }^{\mathrm{d}}$ Department of Surgery and Science, Graduate School of Medical Sciences, Kyushu 
1 Corresponding author and requests for reprints:

2 Yoshinao Oda MD, $\mathrm{PhD}$,

3 Department of Anatomic Pathology, Pathological Sciences,

4 Graduate School of Medical Sciences, Kyushu University,

5 Maidashi 3-1-1, Higashi-ku, Fukuoka 812-8582, Japan

$6 \quad$ E-mail: oda@ surgpath.med.kyushu-u.ac.jp

$7 \quad$ Tel: +81-92-642-6061, FAX: +81-92-642-5968

8

$9 \quad$ Running title: Molecular characteristics of colorectal neuroendocrine carcinoma

\section{Disclosures:}

11 The authors declare that there are no conflicts of interest or funding disclosures to make. 
1 Abstract

2 To further clarify the molecular features of colorectal neuroendocrine carcinomas (NECs), we

3 immunohistochemically examined tumor samples from 25 NECs, including 9 small cell

4 NECs (SCNECs) and 16 large cell NECs (LCNECs), 20 neuroendocrine tumors (NETs), and

521 poorly differentiated adenocarcinomas (PDCs) for the expression of several biomarkers

6 (p53, $\beta$-catenin, Bcl-2, Rb, p16, p21, cyclinD1, and cyclinE), and used sequencing analysis to

7 identify gene alterations of TP53, APC, CTNNB1, KRAS, and BRAF.

8 The frequencies of aberrant p53 expression (88\%), $\beta$-catenin nuclear expression (48\%), and

9 high expression of cyclinE (84\%) were significantly higher in NECs than in NETs $(0 \%, 5 \%$,

10 and $5 \%, \mathrm{P}<.01$, respectively). The immunohistochemical results of NECs and PDCs were

11 similar. TP53, APC, KRAS, and BRAF gene mutations were variously detected in NECs and

12 PDCs but not in any NETs.

The frequencies of decreased expression of $\mathrm{Rb}(56 \%)$ and of high expression of $\mathrm{p} 16(56 \%)$ and Bcl-2 (64\%) were significantly higher in NECs than in PDCs $(5 \%, 19 \%$, and 5\%, P<.05,

15 respectively) or NETs $(10 \%, 5 \%$, and $5 \%, \mathrm{P}<.01$, respectively).

Such immunohistochemical characteristics of NECs were more evident in SCNECs than in

$17 \quad$ LCNECs $(P<.01)$.

18 In conclusion, the molecular features of colorectal NECs are similar to those of

19 adenocarcinomas and not to those of NETs. Decreased expression of Rb and high expression 
1 of p16 and Bcl-2, are characteristics of NECs, suggesting that $\underline{\mathrm{Rb}-\mathrm{p} 16 \text { pathway disruption }}$

2 may contribute to the promotion of proliferative activity in colorectal NECs. SCNECs may be

3 a prototype of NECs.

4

5 Key words: colorectal neuroendocrine carcinoma; neuroendocrine tumor; adenocarcinoma;

6 Rb-p16 pathway; molecular characteristics

7

8 


\section{1. Introduction}

2 Colorectal neuroendocrine carcinomas (NECs) are rare but highly aggressive neoplasms [1-7].

3 A recent WHO classification [1] graded neuroendocrine neoplasms (NENs) into three groups

4 of neuroendocrine tumors-NETs G1, NETs G2, and NECs - simply on the basis of tumor

5 proliferative activity. Morphologically, colorectal NECs are a heterogeneous group ranging

6 from small cell NECs (SCNECs) to large cell NECs (LCNECs). Some cases of LCNECs are

7 difficult to distinguish from poorly differentiated adenocarcinomas (PDCs) with solid growth

8 patterns [7].

9 The mechanisms of carcinogenesis and aggressiveness of colorectal NECs are still largely

10 unknown. Although the expression of some biomarkers and the molecular features of NECs

11 were previously analyzed, only a small number of colorectal NECs were included in most of

12 those papers [8-13].

There is a hypothesis that they are derived from preceding adenoma/adenocarcinomas. This is supported by combined cases with conventional adenoma/adenocarcinoma and NECs, and some molecular features such as the identical loss of heterozygosity [12] or identical mutation [13] of some genes in both components. Aberrant expression of p53 was observed in about $80 \%$ of colorectal NECs in the previous reports $[5,6]$. The expression of other p53-related proteins, such as p21, cyclinE, and Bcl-2, hasen't been clearly described yet in colorectal NECs. 
1 Disruption of the Rb-p16 pathway, which is another key role in the cell-cycle checkpoint, was

2 previously reported in pulmonary and gastrointestinal NECs [8,14]. Overexpression of p16

3 was reported in gastrointestinal NECs, including 6 cases of colorectal NECs [8]. On the other

4 hand, low expression of p16 and the methylation of the CDKN2A gene were reported to be

$5 \quad$ associated with poorer prognosis in some NENs, including colorectal NECs [9].

6 The Wnt- $\beta$-catenin pathway and the expression of cyclinD1 in NENs of various organs have

7 been investigated $[8,10,15,16]$, but colorectal NECs have not been the focus of attention.

8 In this study, we have attempted to sharpen our understanding of the molecular features of

9 colorectal NECs systematically by analyzing a relatively large number of cases and by

10 directly comparing the characteristics of colorectal NECs with those of NETs and PDCs.

\section{Materials and Methods}

\subsection{Patient selection}

14 We first searched the institutional database of the Department of Anatomic Pathology of Kyushu University (Fukuoka, Japan) and related facilities to identify cases diagnosed between 1986 and 2013 as colorectal "neuroendocrine carcinoma", "endocrine cell carcinoma", "small cell carcinoma", and "carcinoma with neuroendocrine differentiation (or features)". We reviewed hematoxylin-eosin-stained sections of all the cases. 
1 WHO classification [1] and a previous report [7]. SCNECs were characterized by sheets or

2 nests of relatively small to medium-sized cells with high nucleus-to-cytoplasm ratios,

3 hyperchromatic nuclei with finely granular chromatins, inconspicuous nucleoli, frequent

4 nuclear moldings, and high mitotic activity; and by necrosis [7]. Positive staining for

$5 \quad$ neuroendocrine markers was not required for diagnosis.

6 LCNECs were characterized by diffuse growth patterns or a "neuroendocrine architecture"

7 (i.e., organoid or nested structures, trabeculae, peripheral palisading, or rosettes) and

8 composed of monotonous round to oval cells with moderate amounts of cytoplasm,

9 granular/vesicular nuclei, visible nucleoli, and high mitotic activity [7]. Necrosis was also

10 common. To confirm diagnoses of LCNECs, we required immunohistochemical positivity for

11 chromogranin A and/or synaptophysin in $>20 \%$ of the tumor area [7]. The mitotic rate was

12 determined by counting 10 high-power fields (HPF). The Ki-67 labeling index (L.I.) was

13 calculated by manually counting Ki-67-positive nuclei among 1000 tumor cells at a hot spot.

14 We carefully excluded metastatic cases from other organs such as the lung.

15 This study was approved by the institutional review board of Kyushu University

16 (IRB\#25-191).

\subsection{Clinical assessment}

18 The clinical characteristics of all cases were estimated, including age, sex, tumor location,

19 tumor size, the presence or absence of nodal/distant metastasis, and tumor stage $[17,18]$. 


\section{$1 \quad 2.3$ Histological assessment}

2 We estimated histological findings including necrosis, mitotic counts, lymphovascular

3 invasion, tumor-infiltrating lymphocytes, focal squamous differentiation, intracytoplasmic

4 mucin, and the presence or absence of additional adenoma/adenocarcinoma components

5 according to the previous reports $[4,5,7,19]$. With regard to the 2 cases for which only biopsy

6 specimens were available, the analyses for lymphovascular invasion, squamous differentiation,

$7 \quad$ and the presence or absence of additional non-NEC components could not be performed.

\section{$8 \quad 2.4$ Immunohistochemical assessment}

9 Representative formalin-fixed and paraffin-embedded (FFPE) blocks were cut into 4- $\mu$ m-thick slices. The antibodies utilized are summarized in Supplementary table 1. For this

11 staining we used a polymer-based detection system (Envision+; Dako, Carpinteria, CA, USA).

12 After deparaffinization, rehydration, inhibition of endogenous peroxidase, and antigen retrieval, sections were exposed to the primary antibodies. After incubation of the secondary antibody, the sections were incubated in 3,3'-diaminobenzidine and counterstained with

15 hematoxylin.

16 We counted the proportion of positive cells (labeling index, \%:L.I.) for each antibody and

17 defined the cutoff between high and low expression by reference to previous reports 
1 pattern or virtually null pattern by reference to a previous study [21]. It is noteworthy that

2 complete negative p53 staining of NET G1 was not interpreted as "aberrant", as the absence

3 of a p53 staining pattern in benign or indolent growth tumors may simply reflect quiescent

4 cells rather than an underlying TP53 gene mutation.

$5 \quad$ Positive and negative controls played appropriate roles.

6

7

\subsection{Mutational analysis}

Tumor DNA of each case was obtained from FFPE blocks. With regard to NETs and NECs, the tumor cells were selectively isolated by laser capture microdissection using a Leica AS

LMD (Leica Microsystems, Tokyo, Japan). For the combined NECs, we separately isolated tumor cells from NECs and adenoma/adenocarcinoma components. With regard to PDCs, we prepared sections of histological areas having high densities of tumor cells so as to minimize the contamination of normal tissues adjacent to tumor cells. Subsequently, genomic DNA was extracted using a QIAamp DNA Micro Kit and a QIAamp DNA FFPE Tissue Kit (Qiagen, Tokyo, Japan), according to the manufacturer's protocols.

We assessed the mutational status of TP53, APC, CTNNB1, KRAS, and BRAF using polymerase chain reaction (PCR) amplification and direct sequencing. Primer sequences and conditions of PCR are summarized in Supplementary table 2.

\subsection{Statistical analysis}

We assessed statistical differences between the groups using the Mann-Whitney U-test, the 
1 Chi-square test, or Fisher's exact test. We investigated associations among the L.I.s of the

2 antibodies by the Spearman rank correlation coefficient test. All calculations were performed

3 using JMP software version 9.0.2 (SAS, Cary, NC, USA). A $P$-value less than .05 was

4 considered statistically significant.

5

6 3. Results

$7 \quad 3.1$ Patient selection

8 We identified 25 cases of colorectal NECs (9 cases of SCNECs and 16 cases of LCNECs)

9 (Fig. 1A-1D). Twenty-three were surgically resected specimens and two were biopsy

10 specimens.

11 We also subclassified them, except for the 2 biopsy cases, into 8 combined NECs (Fig. 1C,

12 D), which had additional adenoma and/or adenocarcinoma components, and 15 pure NECs.

13 Among the combined NECs, 4 tumors fulfilled the criteria of mixed adeno-neuroendocrine

14 carcinoma (MANEC) defined by the WHO classification [1].

15 We also randomly selected 20 cases of NET from the database (Fig. 1E). NETs in this study

16 were characterized according to WHO classification and corresponded to NET G1 (18 cases)

17 and NET G2 (2 cases) [1]. The 2 cases of NET G2 were graded by Ki-67 L.I. (5\% and 4.5\%,

18 respectively) based on the definition in the WHO classification [1].

19 For the control, we randomly selected 21 cases showing predominant solid growth patterns of 
1 PDCs (Fig. 1F). All PDCs were selected after the careful exclusion of neuroendocrine

2 differentiation and hepatoid morphology/differentiation.

\subsection{Clinical characteristics}

4 The clinical characteristics are summarized in Table 1.

5 Compared to patients with NETs, those with NECs were significantly older age (median 68y,

$6 P=.03$ ) and had a larger tumor size (median $5.5 \mathrm{~cm}, P<.0001)$, a higher frequency of lymph

7 node metastasis $(82 \%, P=.0005)$, and a higher frequency of distant metastasis $(44 \%, P=.0005)$,

8 and were at a more advanced stage (Stages III and IV 92\%, $P<.001$ ).

9 No significant differences in clinical characteristics were observed between NECs and PDCs,

10 except for sex distribution $(P=.01)$.

11 NETs showing larger tumor size $(\geqq 3 \mathrm{~cm})$, advanced stage, and lymph node metastasis

12 corresponded to NET G2.

\subsection{Histological characteristics}

14 The results of histological characteristics are summarized in Table 1.

Compared to NETs, NECs showed significantly more frequent lymphatic permeation (NECs

$65 \%, P<.0001$ ), venous invasion (NECs $83 \%, P<.0001$ ), and necrosis (NECs $84 \%, P<.0001$ ).

17 The frequency of the presence of intracytoplasmic mucin and that of tumor-infiltrating 
1 Mitotic counts were significantly higher in NECs (median 29/10HPF) than in NETs (median

$20 ; \mathrm{P}<.0001)$ or PDCs (median 12; $\mathrm{P}=0.01)$.

3 Some NECs coexisted with adenoma and/or adenocarcinoma, but none coexisted with NETs.

4 We found no significant histological differences between SCNECs and LCNECs.

$5 \quad$ In the NETs, venous invasion and mitotic activity were seen only in cases of NET G2.

$6 \quad 3.4$ Immunohistochemical characteristics

$7 \quad$ Immunohistochemical results are summarized in Table 2.

8 Representative immunohistochemical images are shown in Fig. 2.

$9 \quad$ The differences and similarities of molecular characteristics among NECs, NETs, and PDCs.

10 Compared to NETs, NECs showed significantly higher frequencies of aberrant p53 (NECs

$1188 \%, P<.0001$ ) and $\beta$-catenin nuclear expression (NECs 48\%, $P=.0016$ ) and higher

12 expression of cyclinE (NECs $84 \%, P<.0001)$

13 With regard to the expression of those markers, there were no statistical differences between

$14 \quad$ NECs and PDCs.

15 Immunophenotypes in NET G1 were similar to those in NET G2.

16 Molecular characteristics of NECs.

17 NECs showed significantly higher frequencies of decreased expression of $\mathrm{Rb}$ and of high

18 expression of p16 and Bcl-2 than NETs $(P=.0014 ; P=.0003 ; P<.0001$, respectively $)$ or PDCs

$19 \quad(P=.0002 ; P=.0105 ; P<.0001$, respectively $)$. 
1 These characteristic molecular features of NECs (loss of Rb expression and high expression

2 of p16 and Bcl-2) were shown even in the 2 cases of NEC diagnosed on biopsy.

$3 \quad$ The differences and similarities of molecular characteristics between SCNECs and LCNECs.

4 All the SCNECs showed a complete loss of Rb protein and high expression of p16 and Bcl-2.

5 The frequencies of loss of $\mathrm{Rb}$ expression and of high expression of $\mathrm{p} 16$ and $\mathrm{Bcl}-2$ were

6 significantly higher in SCNECs than in LCNECs $(P=.0009 ; P=.0009 ; P=.0049$, respectively).

$7 \quad$ The expression levels of Rb, p16, and Bcl-2 of LCNECs were intermediate between those of

$8 \quad$ SCNECs and those of PDCs (Table 2).

$9 \quad$ The molecular characteristics of NEC components in combined NECs.

10 Detailed immunohistochemical findings of combined NECs are shown in Table 3. Decreased

11 expression of $\mathrm{Rb}$ and high expression of p16 and Bcl-2 were evident in most combined NECs

12 (Table 3, Fig. 2). In half of the combined NECs, $\beta$-catenin nuclear expression was detected

13 only in the NEC components.

14 Correlations of expression levels of molecular markers of NECs.

15 There were significant correlations between decreased expression of $\mathrm{Rb}$ and high expression

16 of p16 in NECs, $(\underline{P=.0002})$, between nuclear $\beta$-catenin expression and high expression of

17 cyclinD1 in SCNECs $(\underline{P=.0128})$, between decreased expression of $\mathrm{Rb}$ and high expression of

18 cyclinD1 in NECs $(P<.0001)$ and LCNECs $\underline{(P=.0295)}$, and between high expression of p21

19 and high expression of cyclinE in SCNECs $(\underline{P=.0246)}$. 
1 No significant correlations were found between the immunohistochemical expression of these

2 markers and the clinicopathological characteristics of NECs.

\subsection{Genetic analysis}

Results of mutational analyses are summarized in Tables 4 and 5 and in Fig. 3.

TP53, APC, KRAS, and BRAF gene mutations were variously observed in NECs and PDCs,

but not in any NETs. Nearly all the tumors with TP53 gene mutation showed an aberrant p53

staining pattern (Table 5). In 2 cases of combined LCNEC, identical TP53 gene mutations were confirmed in both NEC and adenocarcinoma components (Fig. 3A). In 1 case of combined SCNEC, identical KRAS gene mutation was confirmed in NEC, adenoma, and

10 adenocarcinoma components (Fig. 3B). $\underline{\text { mutations were observed in a small population of NETs, NECs, and PDCs (Table 4). }}$

\section{Discussion}

In this study, we sharpened our understanding of the molecular features of colorectal NECs by demonstrating their differences from, and similarities to, those of NETs and PDCs.

17 patterns of several biomarkers, including $\mathrm{p} 53$, cyclinE, and $\beta$-catenin. We demonstrated that 
1 cyclinE in NECs were similar to those in PDCs and higher than those in NETs. High

2 expression of cyclinE, as well as aberrant p53 and nuclear $\beta$-catenin expression, appear to be

3 common features of colorectal NECs and PDCs but not of NETs. We showed similar patterns

4 of genetic alteration between NECs and PDCs. By sequencing analysis, TP53, APC, KRAS,

5 and BRAF mutations were variously observed in NECs and PDCs but not in NETs.

6 In this study, NECs sometimes coexisted with adenoma and/or adenocarcinoma but, as

$7 \quad$ reported previously [5-7], NECs did not coexist with any NETs.

8 Immunohistochemical staining patterns of p53, p21, and cyclinE were also similar in both

$9 \quad$ NEC and adenoma/adenocarcinoma components in combined NECs (Table 3).

10 By sequencing analysis, identical mutations of the KRAS or TP53 gene were demonstrated in

11 both NEC and adenoma/adenocarcinoma components in a few combined NECs.

12 These immunohistochemical and molecular genetic features strongly substantiate the general concept that the molecular characteristics of colorectal NECs are similar to those of

14 adenocarcinomas but not to those of NETs.

15 On the other hand, we demonstrated that low expression of Rb and high expression of $\mathrm{p} 16$

16 and Bcl-2 were frequent in colorectal NECs. We also demonstrated a significant and inverse

17 relationship between $\mathrm{Rb}$ and $\mathrm{p} 16$ expression levels in colorectal NECs.

18 Low expression of $\mathrm{Rb}$ and high expression of $\mathrm{p} 16$ were previously reported in NECs of the

19 lung [14] and gastrointestinal tract, including a small number of colorectal ones [8]. Thus, it 
1 has been speculated that $\mathrm{Rb}-\mathrm{p} 16$ pathway disruption may contribute to the promotion of

2 proliferative activity in NECs. Our results further substantiate the fact that Rb-p16 disruption

3 can explain the high proliferative activity of colorectal NECs. Recently, Rosa et al. analyzed

4 the methylation profiles of 39 cases of colorectal NECs and rarely detected the methylation of

$5 \quad C D K N 2 A$ [5]. This finding is in line with our results, showing high p16 expression.

6 Bcl-2 expression of colorectal NECs has not been specifically investigated previously.

7 Yachida et al. reported Bcl-2 overexpression in $73.6 \%$ of pancreatic NECs [23]. Based on the

8 results of our study and Yachida's report [23], high expression of Bcl-2 may be a

9 characteristic of NECs of both the pancreas and colorectum.

10 In this study, both the loss of Rb expression and the high expression of p16 and Bcl-2 were

11 much more evident in SCNECs than in LCNECs. Surprisingly, tumor cells completely lost Rb

12 expression in all SCNECs. High expression of p16 and Bcl-2 was also evident in all SCNEC

13 cases. In previous reports of the pancreatic and pulmonary NECs [14,23] as well as in our

14 results, these molecular characteristics of NECs were also much more evident in SCNECs

15 than in LCNECs, although the complete loss of Rb expression in SCNECs has not been

16 clearly mentioned until now.

17 On the other hand, Rb/p16/Bcl-2 expression levels in LCNECs were intermediate between

18 SCNECs and PDCs (Table 2). 
1 In this study, patients with NECs showed the statistically shortest overall survival period

2 among NECs, NETs, and PDCs (data not shown). No significant prognostic difference was

3 seen in between SCNECs and LCNECs. From the standpoint of prognosis, SCNECs and

$4 \quad$ LCNECs can be unified into a high-grade category. However, from the standpoint of

5 pathological classification, the morphological features of SCNECs and LCNECs differ

6 significantly by definition. Furthermore, there are some differences in molecular features

7 between them. In this context, we believe that SCNECs and LCNECs should remain separate

8 pathological categories.

$9 \quad$ Furthermore, we believe that LCNECs should not be readily incorporated into PDCs, because

10 LCNECs show 1) unequivocal neuroendocrine morphology and immunophenotype in contrast

11 to PDCs, and 2) significantly worse prognosis than PDCs (data not shown), even though

$12 \quad$ LCNECs have some similar molecular features with PDCs.

In this study, the frequencies of high expression of $\mathrm{Bcl}-2$ and of the loss of $\mathrm{Rb}$ nuclear expression were significantly higher in LCNECs than in PDCs. We further examined the bioptic samples taken from 4 cases of LCNECs and 9 cases of PDCs, for the expression of $\underline{\mathrm{Bcl}-2}$ and Rb. Unfortunately, we did not observe high expression of Bcl-2 or the loss of $\mathrm{Rb}$

17 expression in any LCNECs or in most PDCs $(0 / 9,1 / 9$, respectively). The results of these

18 bioptic samples were mostly concordant with those of surgical specimens. In this context, we

19 think there is limited diagnostic utility of Bcl-2 and Rb immunohistochemistry for the 
1 differential diagnosis between LCNECs and PDCs, since most PDCs and more than half of

$2 \quad$ LCNECs lack high expression of Bcl-2 and loss of Rb expression (Table 2).

$3 \quad$ We think that the careful examination of morphological features on HE slides and the

4 confirmation of neuroendocrine immunophenotype (positive staining of chromogranin A or

5 synaptophysin) are paramount for the differential diagnosis between LCNECs and PDCs.

6 It has been reported that an aberrant $\mathrm{Wnt} / \beta$-catenin pathway was related to the pathogenesis

7 and the progression of NENs of various organs $[6,10,15]$. In the present study, half of the

8 combined NECs showed nuclear $\beta$-catenin expression only in NEC components, suggesting

9 that Wnt pathway activation is also related to neuroendocrine differentiation in some

10 colorectal NECs.

11 In conclusion, the molecular features of colorectal NECs are similar to those of

12 adenocarcinoma and not to those of NETs.

$13 \mathrm{Rb}-\mathrm{p} 16$ pathway disruption and Bcl-2 overexpression may be characteristics of colorectal

14 NECs. SCNECs may be a prototype of NECs, whereas the molecular features of LCNECs are

15 in between those of SCNECs and those of PDCs.

\section{Acknowledgment}

17 The English usage in this article was reviewed by KN International 


\section{Reference}

[1] Klimstra DS, Capella C, Arnold R, et al. Neuroendocrine neoplasms of the colon and rectum. In: Bosaman FT, et al, ed. WHO classification of tumors of the digestive system. Lyon, France: IARC Press;2010:174-177.

[2] Bernick PE, Klimstra DS, Shia J, et al. Neuroendocrine carcinomas of the colon and rectum. Dis Colon Rectum 2004;47:163-169.

[3] Wick MR, Vitsky JL, Ritter JH, et al. Sporadic medullary carcinoma of the colon: a clinicopathologic comparison with nonhereditary poorly differentiated enteric-type adenocarcinoma and neuroendocrine colorectal carcinoma. Am J Clin Pathol 2005;123:56-65.

[4] Cheng CC, Wang CW, Changchien CR, et al. Neuroendcrine Carcinomas of the Colon and Rectum: Result of a 15-year Experience. J Soc Colon Rectal Surgeon 2008;19:87-94.

[5] La Rosa S, Marando A, Furlan D,et al. Colorectal poorly differentiated neuroendocrine carcinomas and mixed adenoneuroendocrine carcinomas: insights into the diagnostic immunophenotype, assessment of methylation profile, and search for prognostic markers. Am J Surg Pathol 2012;35:601-611.

[6] Li Y, Yau A, Schaeffer D, et al. Colorectal glandular-neuroendocrine mixed tumor: pathologic spectrum and clinical implications. Am J Surg Pathol 2011;35:413-425.

[7] Shia J, Tang LH, Weiser MR, et al. Is nonsmall cell type high-grade neuroendocrine carcinoma of the tubular gastrointestinal tract a distinct disease entity? Am J Surg Pathol 
2008;32:719-731.

[8] Li AF, Li AC, Tsay SH, et al. Alterations in the p16INK4a/cyclin D1/RB pathway in gastrointestinal tract endocrine tumors. Am J Clin Pathol 2008;130:535-542.

[9] Simon B, Lubomierski N. Implication of the INK4a/ARF locus in gastroenteropancreatic neuroendocrine tumorigenesis. Ann N Y Acad Sci 2004;1014:284-299.

[10] Pizzi S, Azzoni C, Tamburini E, et al. Adenomatous polyposis coli alteration in digestive endocrine tumours: correlation with nuclear translocation of beta-catenin and chromosomal instability. Endocr Relat Cancer 2008;15:1013-1024.

[11] Stelow EB, Moskaluk CA, Mills SE. The mismatch repair protein status of colorectal small cell neuroendocrine carcinomas. Am J Surg Pathol 2006;30:1401-1404.

[12] Vortmeyer AO, Lubensky IA, Merino MJ, et al. Concordance of genetic alterations in poorly differentiated colorectal neuroendocrine carcinomas and associated adenocarcinomas. J Natl Cancer Inst 1997;89:1448-1453.

[13] Karkouche R, Bachet JB, Sandrini J, et al. Colorectal neuroendocrine carcinomas and adenocarcinomas share oncogenic pathways. A clinico-pathologic study of 12 cases. Eur J Gastroenterol Hepatol. 2012;24:1430-1437.

[14] Beasley MB, Lantuejoul S, Abbondanzo S, et al. The P16/cyclin D1/Rb pathway in neuroendocrine tumors of the lung. Hum Pathol 2003;34:136-142.

[15] Kim JT, Li J, Jang ER, et al. Deregulation of Wnt/ $\beta$-catenin signaling through genetic or 
1 epigenetic alterations in human neuroendocrine tumors. Carcinogenesis 2013;34:953-961.

2 [16] Fujimori M, Ikeda S, Shimizu Y, et al. Accumulation of beta-catenin protein and

3 mutations in exon 3 of beta-catenin gene in gastrointestinal carcinoid tumor. Cancer Res

$4 \quad 2001 ; 61: 6656-6659$.

5 [17] Sobin LH, Gospodarowicz MK, Wittekind CTNM. Classification of Malignant Tumours.

$6 \quad 7^{\text {th }}$ ed. Oxford, UK: Wiley-Blackwell; 2009.

7 [18] Rindi G, Kloppel G, Couvelard A, et al. TNM staging of midgut and hindgut

8 (neuro)endocrine tumors: a consensus proposal including a grading system. Virchows Arch.

$9 \quad 2007 ; 451: 757-762$.

10 [19] Jenkins MA, Hayashi S, O'Shea AM, et al. Pathology features in Bethesda guidelines

11 predict colorectal cancer microsatellite instability: a population-based study. Gastroenterology

$12 \quad 2007 ; 133: 48-56$.

13 [20] Gasparini G, Barbareschi M, Doglioni C, et al. Expression of bcl-2 protein predicts

14 efficacy of adjuvant treatments in operable node-positive breast cancer. Clin Cancer Res.

$15 \quad 1995 ; 1: 189-198$.

16 [21] Yemelyanova A, Vang R, Kshirsagar M, et al. Immunohistochemical staining patterns of

17 p53 can serve as a surrogate marker for TP53 mutations in ovarian carcinoma: an

18 immunohistochemical and nucleotide sequencing analysis. Mod Pathol 2011;24:1248-1253.

19 [22] Zhou Z, Bandla S, Ye J, et al. Cyclin E involved in early stage carcinogenesis of 
1 esophageal adenocarcinoma by SNP DNA microarray and immunohistochemical studies.

$2 \quad$ BMC Gastroenterol 2014;14:78.

3 [23] Yachida S, Vakiani E, White CM, et al. Small cell and large cell neuroendocrine

4 carcinomas of the pancreas are genetically similar and distinct from well-differentiated

5 pancreatic neuroendocrine tumors. Am J Surg Pathol 2012;36:173-184.

6

7 


\section{$1 \quad$ Titles and legends to figures}

2 Figure 1. Representative histological findings of colorectal neuroendocrine carcinoma.

$3 \quad$ A, LCNEC with neuroendocrine architecture.

4 B, SCNEC with nested growth pattern and peripheral palisading.

5 C, Combined NEC with gland-forming adenocarcinoma and SCNEC.

6 D, Combined NEC with gland-forming adenocarcinoma and LCNEC.

7 E, NET G1 with trabecular growth pattern and focal rosette structure.

8 F, PDC showing solid nests.

9 (Magnifications; A-Fx200)

10 Figure 2. Representative images of immunohistochemical staining.

$11 \mathrm{~A}, \mathrm{Rb}$ expression in combined SCNEC. Complete loss of nuclear staining was seen in

12 SCNEC component (lower). Rb expression was retained only in the adenocarcinoma 13 component (upper).

14 B, p16 expression in combined SCNEC. Diffuse nuclear and cytoplasmic staining was seen in

15 SCNEC component (left). Adenocarcinoma component showed scattered staining.

16 C, Bcl-2 expression in combined SCNEC. Diffuse cytoplasmic staining was seen in SCNEC

17 component (left). Adenocarcinoma component showed negative staining (right).

D, p53 expression in LCNEC. Diffuse nuclear staining is visible.

E, p21 expression in LCNEC. Diffuse nuclear staining is visible. 
1 F, CyclinE expression in LCNEC. Diffuse nuclear staining is visible.

$2 \mathrm{G}, \beta$-catenin expression in combined SCNEC. Diffuse nuclear staining was seen in the

3 SCNEC component. Coexisting adenoma component showed membranous staining.

$4 \mathrm{H}$, CyclinD1 expression in combined SCNEC. Both NECs and adenocarcinoma component

$5 \quad$ showed nuclear staining.

6 (Magnifications; A-Ix200)

7 Figure 3. Sequencing results of TP53 and KRAS gene mutation in combined NEC.

8 A, Sequencing results of TP53 gene mutation in combined NECs. (upper) Sequencing results

9 of TP53 gene exon5 in NEC component. Sequencing shows the substitution of CGC to CAC

10 at codon 175 , causing an amino acid change from arginine to histidine. (lower) Identical

11 mutation was shown in associated adenocarcinoma component.

$12 \mathrm{~B}$, Sequencing results of KRAS gene mutation in combined NECs. (upper) Sequencing results 13 of KRAS gene exon2 in NEC component. Sequencing shows the substitution of GGC to GAC

14 at codon 13, causing an amino acid change from glycine to aspartic acid.

15 Identical mutation was detected in additional adenoma (middle) and adenocarcinoma (lower) 16 components.

17 Abbreviations: NEC, neuroendocrine carcinoma; NET, neuroendocrine tumor;

18 PDC, poorly differentiated adenocarcinoma; SCNEC, small cell neuroendocrine carcinoma;

$19 \quad$ LCNEC, large cell neuroendocrine carcinoma 


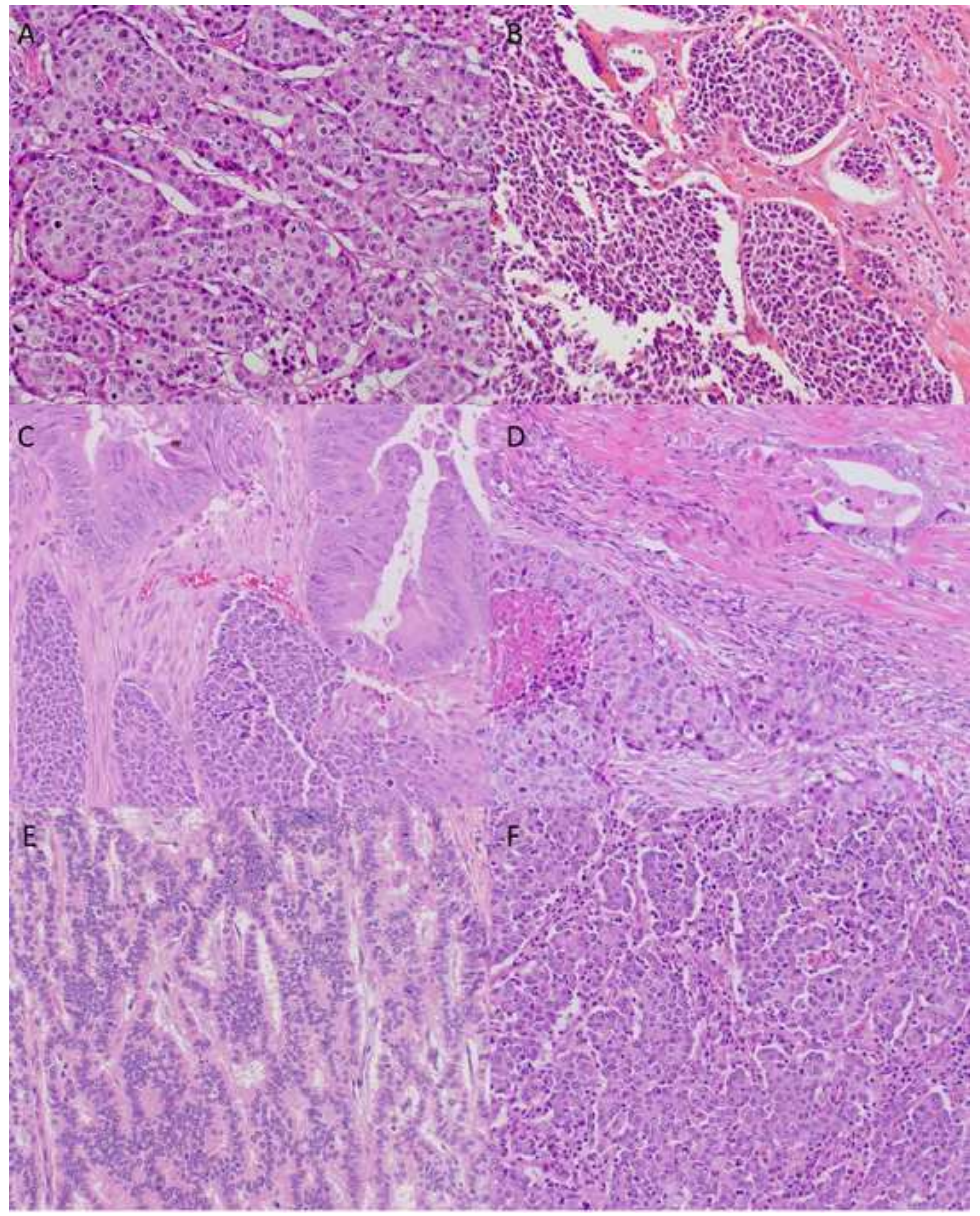


Click here to download high resolution image
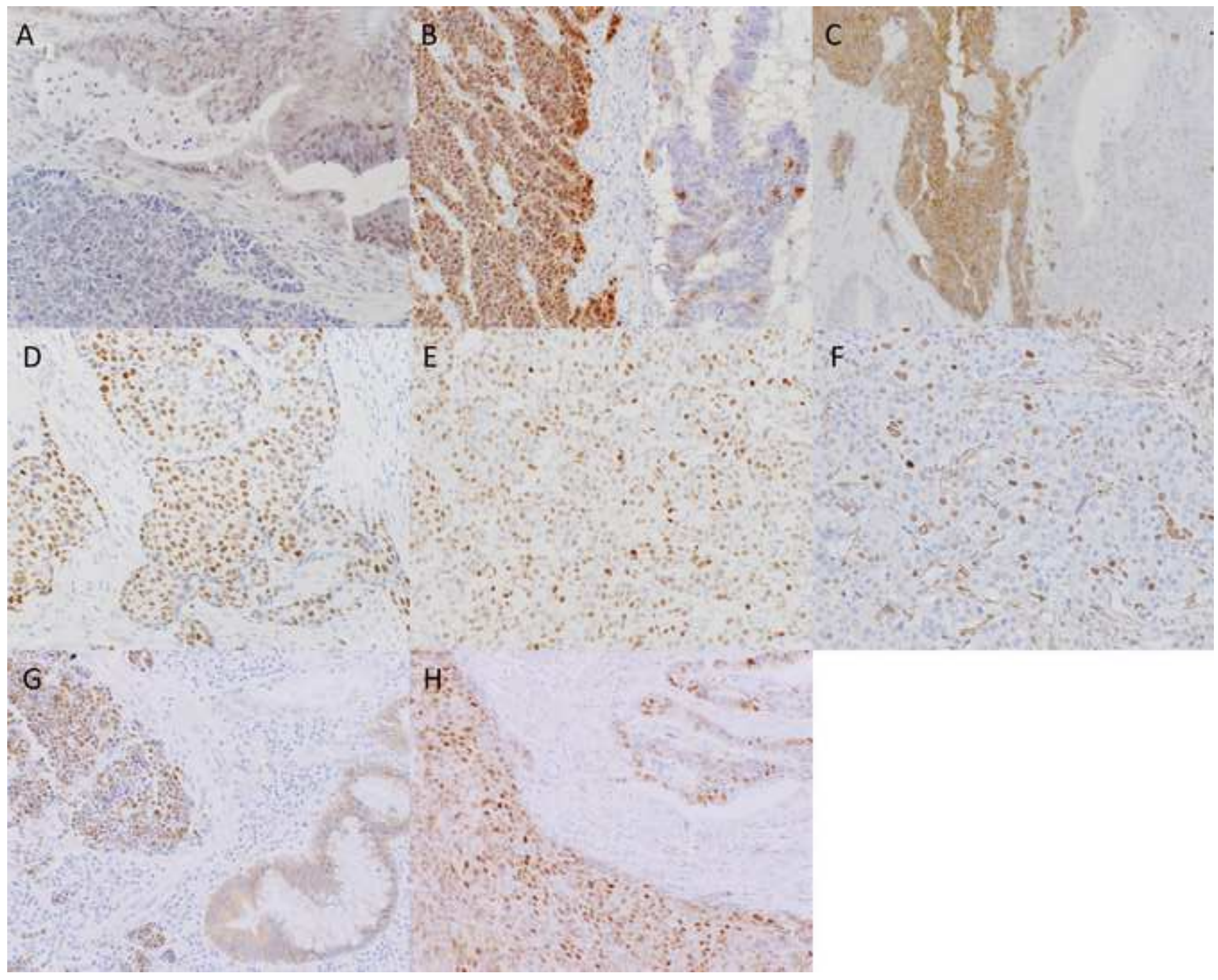
A

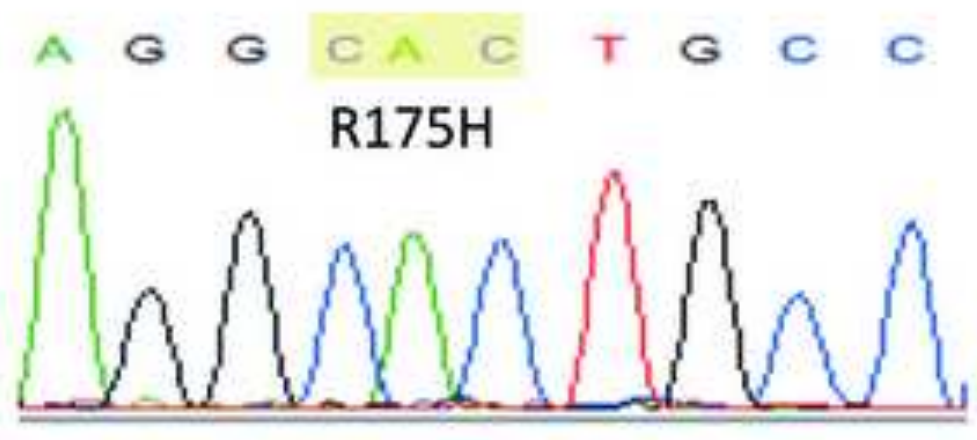

NEC component
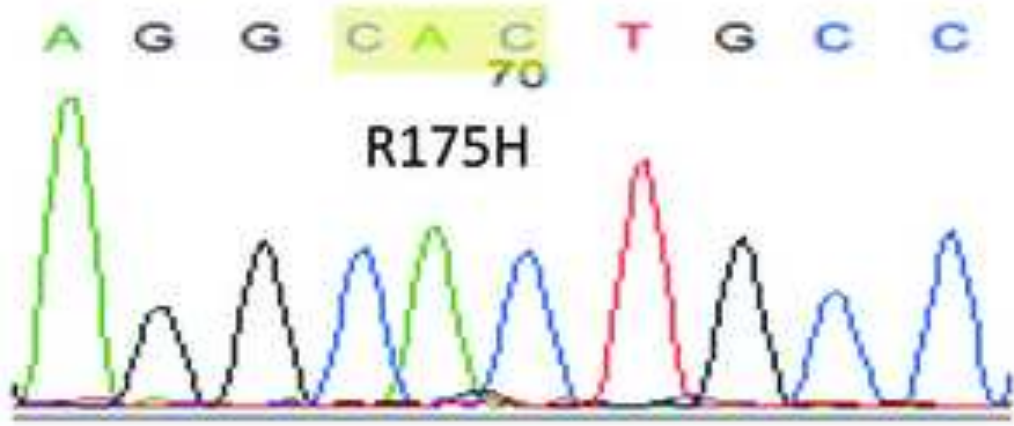

Adenocarcinoma component

B

C $T \underset{20}{G} G$ T G G C G T A G G13D

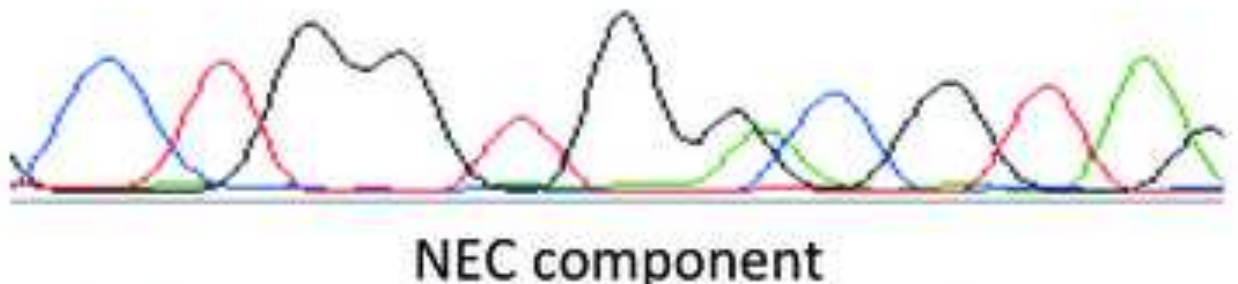

G13D

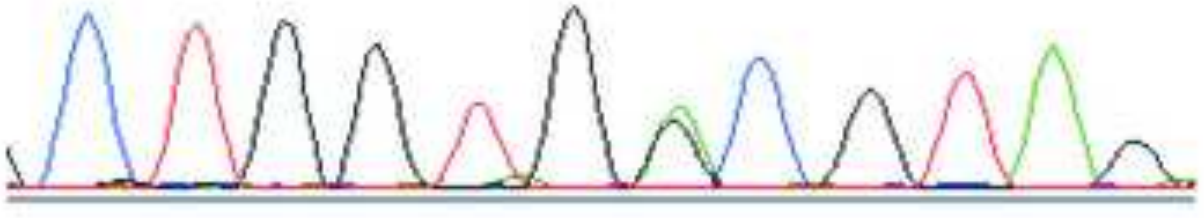

Adenoma component

$\begin{array}{lcllllllllll}C & T & G & G & T & G & N & C & G & T & A & G \\ 110 & & & & & & & & & & & \end{array}$



Adenocarcinoma component 
Table 1. Clinical and histological characteristics of colorectal NECs, NETs, and PDCs

\begin{tabular}{|c|c|c|c|c|c|}
\hline & $\begin{array}{l}\text { NETs } \\
n=20\end{array}$ & $\begin{array}{l}\text { NECs } \\
\mathrm{n}=25\end{array}$ & $\begin{array}{l}\text { PDCs } \\
n=21\end{array}$ & $\begin{array}{l}\text { SCNECs } \\
\mathrm{n}=9\end{array}$ & $\begin{array}{l}\text { LCNECs } \\
\mathrm{n}=16\end{array}$ \\
\hline Age (y), median (range) & $57(24-86)$ & $68(46-88)^{b}$ & $70(33-85)$ & $68(48-73)$ & $70(46-88)$ \\
\hline Sex male/female (\%) & $65 / 35$ & $68 / 32^{\mathrm{C}}$ & $33 / 67$ & $11 / 89$ & $56 / 44$ \\
\hline Tumor location (R/L) $(\%)^{a}$ & $0 / 100$ & $32 / 68^{b}$ & $48 / 52$ & $11 / 89$ & $44 / 56$ \\
\hline Tumor size (cm), median (range) & $0.75(0.2-3.8)$ & $5.5(1.5-16)^{b}$ & $6.7(2.1-12.5)$ & $4.75(2.5-7.0)$ & $6.0(1.5-16)$ \\
\hline Lymph node metastasis (\%) & 10 & $82^{b}$ & 71 & 71 & 87 \\
\hline $\begin{array}{l}\text { Distant metastasis (\%) } \\
\text { Tumor stage (\%) }\end{array}$ & 0 & $44^{b}$ & 33 & 44 & 44 \\
\hline (UICC) Stage I/ II/ III/ IV & $90 / 0 / 10 / 0$ & $0 / 8 / 48 / 44^{b}$ & 0/29/38/33 & 0/22/33/44 & $0 / 0 / 56 / 44$ \\
\hline 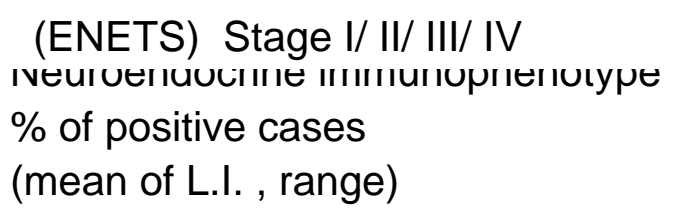 & $90 / 0 / 10 / 0$ & $0 / 8 / 48 / 44^{b}$ & & $0 / 22 / 33 / 44$ & $0 / 0 / 56 / 44$ \\
\hline Chromogranin A & $75(70,0-100)$ & $44(20,0-90)^{b, c}$ & $0(0.5,0-10)$ & $33(22,0-80)$ & $50(19,0-90)$ \\
\hline Synaptophysin & $90(87,0-100)$ & $96(64,0-100)^{c}$ & $0(0.5,0-10)$ & $89(58,0-90)$ & $100(68,20-100)$ \\
\hline Necrosis (\%) & 0 & $84^{b}$ & 62 & 88 & 87 \\
\hline Mitosis /10HPF, median (range) & $0(0-2)$ & $29(2-115)^{b, c}$ & $12(2-54)$ & $23(5-115)$ & $33(2-72)$ \\
\hline Lymphatic permeation (\%) & 0 & $65^{b}$ & 71 & 75 & 67 \\
\hline Venous invasion (\%) & 5 & $83^{b}$ & 62 & 88 & 87 \\
\hline Tumor-infiltrating lymphocytes (\%) & 0 & $8^{c}$ & 33 & 13 & 0 \\
\hline Focal squamous differentiation (\%) & 0 & 13 & 14 & 25 & 7 \\
\hline $\begin{array}{l}\text { Intracytoplasmic mucin (\%) } \\
\text { Additional components (\%) }\end{array}$ & 0 & $16^{\mathrm{c}}$ & 71 & 13 & 20 \\
\hline NETs & - & 0 & 0 & 0 & 0 \\
\hline Adenoma or adenocarcinoma & 0 & $35^{d}$ & - & 50 & $27^{d}$ \\
\hline
\end{tabular}

a: R means right-side colon including "cecum, ascending colon, and right side of transverse colon".

$\mathrm{L}$ means left-side colon including "left side of transverse colon, descending colon, sigmoid colon, and rectum".

b: $P<0.01$, NECs vs NETs

c: $P<0.01$, NECs vs PDCs

d: One case was a signet-ring cells component.

Abbreviations: NEC, neuroendocrine carcinoma; NET, neuroendocrine tumor;

PDC, poorly differentiated adenocarcinoma; SCNEC, small cell neuroendocrine carcinoma;

LCNEC, large cell neuroendocrine carcinoma; UICC, Union for International Cancer Control;

ENETS, European Neuroendocrine Tumor Society; L.I., labeling index; HPF, high-power field 
Table 2. Summary of immunohistochemical results of NECs, NETs, and PDCs

\begin{tabular}{|c|c|c|c|c|c|c|c|c|}
\hline & & $\begin{array}{l}\text { NETs } \\
n=20\end{array}$ & $\begin{array}{l}\text { NECs } \\
n=25\end{array}$ & $\begin{array}{l}\text { PDCs } \\
n=21\end{array}$ & $\begin{array}{c}\text { SCNECS } \\
n=9\end{array}$ & $\begin{array}{c}\text { LCNECs } \\
n=16\end{array}$ & $\begin{array}{l}\text { Pure } \\
\mathrm{n}=15\end{array}$ & $\begin{array}{c}\text { Combined }^{f} \\
n=8\end{array}$ \\
\hline \multirow{4}{*}{$\begin{array}{l}\text { Loss of expression } \\
\text { of } R b\end{array}$} & $\%$ of abnormality & 10 & $56^{\mathrm{ab}}$ & 5 & $100^{c}$ & 31 & 40 & 75 \\
\hline & Mean of L.I. & 79 & $\underline{34}$ & 79 & 0 & $\underline{54}$ & 48 & 16 \\
\hline & Median of L.I. & 90 & $0^{\mathrm{ab}}$ & 80 & $0^{c}$ & 70 & 70 & 0 \\
\hline & Range of L.I. & $10-90$ & $0-90$ & $0-90$ & 0 & $0-90$ & $0-90$ & $0-70$ \\
\hline \multirow{4}{*}{$\begin{array}{l}\text { High expression } \\
\text { of } p 16\end{array}$} & $\%$ of abnormality & 5 & $\underline{56^{\mathrm{ab}}}$ & 19 & $100^{c}$ & $\underline{31}$ & 40 & 75 \\
\hline & Mean of L.I. & 11 & $\underline{59}$ & 23 & 89 & $\underline{42}$ & 47 & 76 \\
\hline & Median of L.I. & 5 & $\underline{80^{a b}}$ & 2 & $95^{c}$ & 40 & 60 & 95 \\
\hline & Range of L.I. & $0-65$ & $0-95$ & $0-90$ & $70-95$ & $0-95$ & $0-95$ & $10-95$ \\
\hline \multirow{4}{*}{$\begin{array}{l}\text { High expression } \\
\text { of bcl-2 }\end{array}$} & $\%$ of abnormality & 5 & $\underline{64^{\mathrm{ab}}}$ & 5 & $100^{c}$ & $\underline{44}$ & 53 & 75 \\
\hline & Mean of L.I. & 4 & $\underline{46}$ & 3 & 69 & $\underline{33}$ & 41 & 46 \\
\hline & Median of L.I. & 0 & $60^{\mathrm{ab}}$ & 0 & 60 & 0 & 50 & 60 \\
\hline & Range of L.I. & $0-90$ & $0-80$ & $0-50$ & $50-90$ & $0-90$ & $0-90$ & $0-90$ \\
\hline \multirow{4}{*}{$\begin{array}{l}\text { Aberrant expression } \\
\text { of p53 }\end{array}$} & $\%$ of abnormality & 0 & $88^{a}$ & 67 & $67^{c}$ & 100 & 87 & 88 \\
\hline & Mean of L.I. & 0.1 & 40 & 36 & 17 & 53 & 49 & 25 \\
\hline & Median of L.I. & 0 & $30^{a}$ & 2 & 0 & 80 & 80 & 0 \\
\hline & Range of L.I. & $0-2$ & $0-95$ & $0-95$ & $0-70$ & $0-90$ & $0-95$ & $0-90$ \\
\hline \multirow{4}{*}{$\begin{array}{l}\text { High expression } \\
\text { of p21 }\end{array}$} & $\%$ of abnormality & $\underline{40}$ & $\underline{68}$ & 81 & 78 & $\underline{63}$ & 67 & 63 \\
\hline & Mean of L.I. & 6 & $\underline{16}$ & 27 & 15 & $\underline{17}$ & 19 & 10 \\
\hline & Median of L.I. & 5 & $12^{b}$ & 26 & 15 & 9 & 11 & 13 \\
\hline & Range of L.I. & $0-16$ & $0-59$ & $0-63$ & $0-35$ & $0-80$ & $0-30$ & $0-20$ \\
\hline \multirow{4}{*}{$\begin{array}{l}\text { High expression } \\
\text { of cyclinE }\end{array}$} & $\%$ of abnormality & 5 & $\underline{84^{a}}$ & 90 & 100 & $\underline{75}$ & 93 & 75 \\
\hline & Mean of L.I & 5 & $\underline{28}$ & 31 & 30 & $\underline{28}$ & 34 & 19 \\
\hline & Median of L.I & 4 & $\underline{28^{a}}$ & 31 & 28 & $\underline{28}$ & $\underline{30}$ & 23 \\
\hline & Range of L.I & $0-15$ & $0-70$ & $0-70$ & $16-53$ & $0-70$ & $1-70$ & $0-32$ \\
\hline \multirow{4}{*}{$\begin{array}{l}\text { Nuclear expression } \\
\text { of } \beta \text {-catenin }\end{array}$} & $\%$ of abnormality & 5 & $48^{a}$ & 38 & 44 & 50 & 47 & 62 \\
\hline & Mean of L.I. & 4 & 31 & 20 & 26 & 34 & 32 & 39 \\
\hline & Median of L.I. & 0 & $5^{a}$ & 5 & 5 & 29 & 0 & 53 \\
\hline & Range of L.I. & $0-42$ & $0-86$ & $0-80$ & $0-66$ & $0-86$ & $0-86$ & $0-76$ \\
\hline \multirow{4}{*}{$\begin{array}{l}\text { High expression } \\
\text { of cyclinD1 }\end{array}$} & $\%$ of abnormality & 100 & $\underline{60^{a b}}$ & 100 & $22^{c}$ & $\underline{81}$ & $80^{d}$ & 38 \\
\hline & Mean of L.I & 73 & $\underline{36}$ & 58 & 7 & $\underline{53}$ & 51 & 18 \\
\hline & Median of L.I & 72 & $\underline{30^{a b}}$ & 60 & $1^{c}$ & $\underline{60}$ & $52^{d}$ & 6.2 \\
\hline & Range of L.I & $58-88$ & $0-83$ & $20-92$ & $0-30$ & $1-88$ & $0-88$ & $0-83$ \\
\hline \multirow[t]{3}{*}{ Ki-67 L.I. } & Mean of L.I. & 1.1 & 57 & 51 & 60 & 56 & 60 & 52 \\
\hline & Median of L.I. & 0.8 & $55^{a}$ & 53 & 60 & 55 & 55 & 56 \\
\hline & Range of L.I. & $0.1-5$ & $25-87$ & $20-79$ & $37-87$ & $25-83$ & $25-87$ & $29-64$ \\
\hline
\end{tabular}

a $P<0.05$, NECs vs NETs

b $P<0.05$, NECs vs PDCs

c $P<0.05$, SCNECs vs LCNECs

d $P<0.05$, Pure NECs vs Combined NECs

e \%abnormality means the percentage of the cases which showed aberrant expression of each antibody.

$f$ Combined NECs were evaluated in the NECs component.

Abbreviations: NEC, neuroendocrine carcinoma; NET, neuroendocrine tumor;

PDC, poorly differentiated adenocarcinoma; SCNEC, small cell neuroendocrine carcinoma; LCNEC, large cell neuroendocrine carcinoma; L.I.,

labeling index 
Table 3. Summary of immunohistochemical results in combined NECs

\begin{tabular}{|c|c|c|c|c|c|c|c|c|}
\hline Case & $\begin{array}{l}\text { Loss of } \\
\text { expression } \\
\text { of } R b\end{array}$ & $\begin{array}{l}\text { High } \\
\text { expression } \\
\text { of } \text { p16 }\end{array}$ & $\begin{array}{l}\text { High } \\
\text { expression } \\
\text { of bcl } 2\end{array}$ & $\begin{array}{l}\text { Aberrant } \\
\text { expression } \\
\text { of p53 }\end{array}$ & $\begin{array}{l}\text { High } \\
\text { expression } \\
\text { of p21 }\end{array}$ & $\begin{array}{l}\text { High } \\
\text { expression } \\
\text { of cyclin E }\end{array}$ & $\begin{array}{l}\text { Nuclear } \\
\text { expression } \\
\text { of } \beta \text {-catenin }\end{array}$ & $\begin{array}{l}\text { High } \\
\text { expression } \\
\text { of cyclinD1 }\end{array}$ \\
\hline 1 SCNEC & + & + & + & - & + & + & - & - \\
\hline Adenocarcinoma & + & + & - & - & + & + & + & - \\
\hline 2 SCNEC & + & + & + & + & + & + & + & + \\
\hline Adenocarcinoma & - & - & - & - & + & + & - & + \\
\hline 3 SCNEC & + & + & + & + & + & + & - & - \\
\hline Adenocarcinoma & - & + & - & + & + & + & - & + \\
\hline 4 LCNEC & + & + & - & + & - & + & + & + \\
\hline Adenocarcinoma & + & + & - & + & - & + & + & + \\
\hline 5 LCNEC & - & - & - & + & - & + & + & + \\
\hline Adenocarcinoma & - & - & - & + & - & + & - & + \\
\hline 6 LCNEC & - & - & + & + & - & - & - & - \\
\hline Signet-ring cells & - & - & - & + & + & - & - & - \\
\hline 7 SCNEC & + & + & + & + & + & + & + & - \\
\hline Tubulovillous adenoma & - & - & - & + & - & + & - & + \\
\hline 8 LCNEC & + & + & + & + & + & - & + & - \\
\hline Tubulovillous adenoma & - & - & - & + & + & - & - & - \\
\hline
\end{tabular}

Abbreviations: NEC, neuroendocrine carcinoma; SCNEC, small cell neuroendocrine carcinoma; LCNEC, large cell neuroendocrine carcinoma 
Table 4. Summary of genetic alterations of NECs, NETs, and PDCs

\begin{tabular}{cccccc}
\hline Gene & $\begin{array}{c}\text { NETs } \\
\mathrm{n}=20\end{array}$ & $\begin{array}{c}\text { NECs } \\
\mathrm{n}=24\end{array}$ & $\begin{array}{c}\text { PDCs } \\
\mathrm{n}=21\end{array}$ & $\begin{array}{c}\text { SCNECs } \\
\mathrm{n}=9\end{array}$ & $\begin{array}{c}\text { LCNECs } \\
\mathrm{n}=15\end{array}$ \\
\hline TP53 & 0 & 5 & $6^{\mathrm{a}}$ & 0 & 5 \\
APC & 0 & 1 & 5 & 1 & 0 \\
CTNNB1 & 1 & 1 & 2 & 0 & 1 \\
KRAS & 0 & 2 & $2^{\mathrm{b}}$ & 1 & 1 \\
BRAF & 0 & 1 & 3 & 0 & 1 \\
\hline
\end{tabular}

a: Not all the exons were successfully sequenced.

In two cases of PDCs, one case was not successfully sequenced for exon 6 and the other was not succesfully sequenced for exon 8.

b: One case was not successfully sequenced.

Abbreviations: NEC, neuroendocrine carcinoma; NET, neuroendocrine tumor;

PDC, poorly differentiated adenocarcinoma; SCNEC, small cell neuroendocrine carcinoma;

LCNEC, large cell neuroendocrine carcinoma 
Table 5. Detailed results of genetic alterations of NECs, NETs, and PDCs

\begin{tabular}{|c|c|c|c|c|c|c|c|c|}
\hline Gene & Exon & Codon & $\begin{array}{l}\text { Nucleotide } \\
\text { change }\end{array}$ & $\begin{array}{l}\text { Mutation } \\
\text { type }\end{array}$ & $\begin{array}{l}\text { Amino acid } \\
\text { change }\end{array}$ & Tissue type & $\begin{array}{l}\text { Aberrant } \\
\text { expression } \\
\text { of p53 }\end{array}$ & $\begin{array}{l}\text { Nuclear } \\
\text { expression } \\
\text { of } \beta \text {-catenin }\end{array}$ \\
\hline \multirow[t]{12}{*}{ TP53 } & 5 & 152 & $\mathrm{C}>\mathrm{T}$ & missense & $\mathrm{P} \rightarrow \mathrm{L}$ & PDC & - & \\
\hline & 5 & 166 & $C>T$ & missense & $S \rightarrow L$ & $\mathrm{PDC}^{\mathrm{a}}$ & - & \\
\hline & & & & & & PDC & + & \\
\hline & 5 & 172 & $\mathrm{~T}>\mathrm{C}$ & missense & $V \rightarrow A$ & $P^{\prime a}$ & - & \\
\hline & 5 & 175 & $G>A$ & missense & $\mathrm{R} \rightarrow \mathrm{H}$ & $\operatorname{NEC}(\text { LCNEC })^{\mathrm{e}}$ & + & \\
\hline & & & & & & $\operatorname{NEC}(\text { LCNEC })^{\mathrm{e}}$ & + & \\
\hline & 6 & 215 & $G>T$ & missense & $S \rightarrow I$ & $\operatorname{NEC}(\text { LCNEC })^{f}$ & + & \\
\hline & 7 & 248 & $C>T$ & missense & $A \rightarrow W$ & $\operatorname{NEC}(\text { LCNEC })^{b}$ & + & \\
\hline & 8 & 273 & $G>A$ & missense & $\mathrm{R} \rightarrow \mathrm{H}$ & PDC & + & \\
\hline & & & & & & PDC & + & \\
\hline & 8 & 276 & $G>A$ & missense & $A \rightarrow T$ & NEC (LCNEC) & + & \\
\hline & 8 & 282 & $\mathrm{C}>\mathrm{T}$ & missense & $\mathrm{R} \rightarrow \mathrm{W}$ & PDC & + & \\
\hline \multirow[t]{9}{*}{$A P C$} & 15 & 1309 & $G>T$ & nonsense & $E \rightarrow X$ & $\mathrm{PDC}^{\mathrm{C}}$ & & + \\
\hline & 15 & 1313 & $A>G$ & missense & $\mathrm{T} \rightarrow \mathrm{A}$ & PDC & & + \\
\hline & 15 & 1316 & $G>A$ & missense & $A \rightarrow T$ & $\operatorname{PDC}^{d}$ & & + \\
\hline & 15 & 1355 & $\mathrm{C}>\mathrm{T}$ & missense & $S \rightarrow F$ & PDC & & - \\
\hline & 15 & 1362 & $C>T$ & silent & $\mathrm{P} \rightarrow \mathrm{P}$ & $P^{\prime d}$ & & + \\
\hline & 15 & 1363 & $\mathrm{C}>\mathrm{T}$ & silent & $S \rightarrow S$ & $\operatorname{PDC}^{d}$ & & + \\
\hline & 15 & 1414 & $G>A$ & missense & $\mathrm{V} \rightarrow \mathrm{I}$ & NEC (SCNEC) & & + \\
\hline & 15 & 1416 & $G>A$ & missense & $\mathrm{G} \rightarrow \mathrm{S}$ & $P^{\prime} C^{c}$ & & + \\
\hline & 15 & 1498 & $G>A$ & missense & $\mathrm{D} \rightarrow \mathrm{N}$ & PDC & & + \\
\hline \multirow[t]{4}{*}{ CTNNB1 } & 3 & 25 & $G>A$ & nonsense & $W \rightarrow X$ & PDC & & - \\
\hline & 3 & 55 & $A>G$ & missense & $\mathrm{E} \rightarrow \mathrm{G}$ & NET G1 & & - \\
\hline & & & & & & PDC & & - \\
\hline & 3 & 57 & $G>A$ & missense & $\mathrm{V} \rightarrow \mathrm{M}$ & $\operatorname{NEC}(\text { LCNEC })^{f}$ & & - \\
\hline \multirow[t]{2}{*}{ KRAS } & 2 & 13 & $G>A$ & missense & $\mathrm{G} \rightarrow \mathrm{D}$ & NEC (SCNEC) $)^{\mathrm{e}}$ & & \\
\hline & 2 & 12 & $G>A$ & missense & $\mathrm{G} \rightarrow \mathrm{D}$ & $\begin{array}{l}\text { NEC (LCNEC) } \\
\text { PDC }\end{array}$ & & \\
\hline$B A R F$ & 15 & 600 & $\mathrm{~T}>\mathrm{A}$ & missense & $\mathrm{V} \rightarrow \mathrm{E}$ & $\begin{array}{l}\text { NEC }(\text { LCNEC })^{b} \\
\text { PDC }\end{array}$ & & \\
\hline
\end{tabular}

Superscript letters a-d each refer to the same case.

e: In Combined NEC, identical mutations are shown in both NECs and additional components.

$\mathrm{f}$ : In two cases of Combined NEC, each mutation was only shown in NECs component.

Abbreviations: NEC, neuroendocrine carcinoma; NET, neuroendocrine tumor;

PDC, poorly differentiated adenocarcinoma; SCNEC, small cell neuroendocrine

carcinoma; LCNEC, large cell neuroendocrine carcinoma

$C$, cytosine; $T$, thymine; $G$, guanine; $A$, adenine

A, Alanine; D, Aspartic acid; E, Glutamic acid; F, Phenylalanine; G, Glycine; H, Histidine; I, Isoleucine; L, Leucine

M, Methionine; N, Asparagine; P, Proline; R, Arginine; S, Serine; T, Threonine; V, Valine; W, Tryptophan; X, stop codon 
Click here to download Supplementary Material: Supplementary Table 1.xls

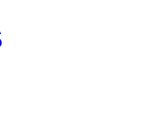

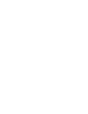

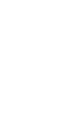
.

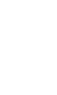

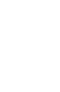





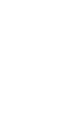

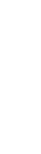
res (1) (1) (1) (1) (1)

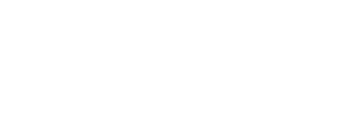

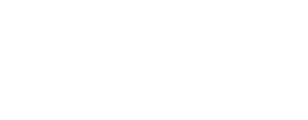

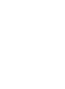
. . . . 
Supplementary Table 2
Click here to download Supplementary Material: Supplementary Table 2.xls

( 